\section{Vorsicht Falle: KV muss nicht mehr an CME-Pflicht erinnern}

_ Die KBV regelt laut $\$ 95 \mathrm{~d}$ Abs. 6 SGB V den Umfang der Fortbildungs-

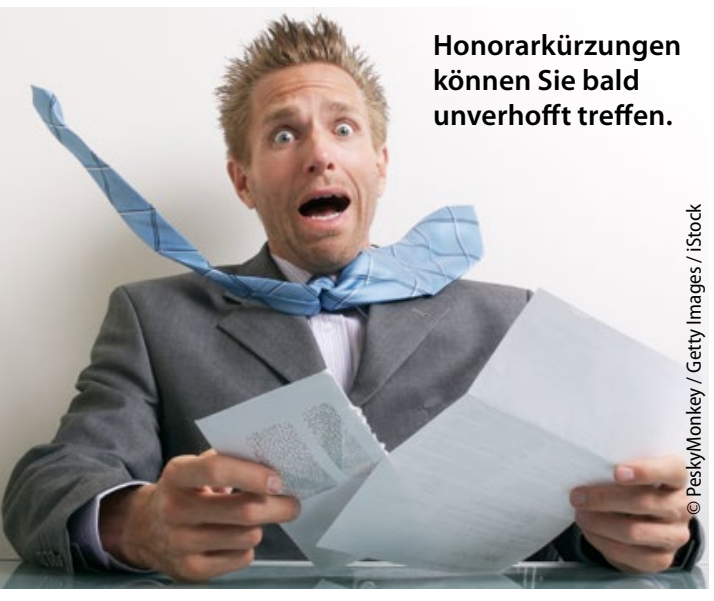

pflicht der Vertragsärzte wie auch das Procedere für den Nachweis und ggf. anstehende Honorarkürzungen. Die Vertreterversammlung (VV) hat nun am 16. September 2016 die Regelungen geändert. Seit Oktober sind die KVen nicht mehr wie bisher verpflichtet, ihre Vertragsärzte und -psychotherapeuten mindestens drei Monate vor Ablauf ihres individuellen Nachweiszeitraums darauf hinzuweisen, dass bei Versäumnis der Frist eine Honorarkürzung gemäß $\$ 95 d$ Abs. 3 Satz 4 SGB V droht.

\section{MMW-KOMMENTAR}

Bisher stand der Servicegedanke bei der Rolle der KVen im Bereich der Fortbildung im Vor-

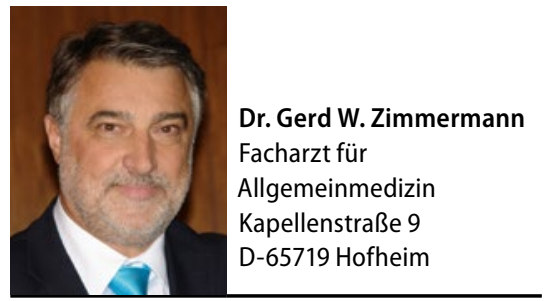

dergrund. Das wurde aus unverständlichen Gründen praktisch fallen gelassen. Die Gefahr, sich bei nicht vollständiger Beachtung der Fortbildungspflicht eine Honorarkürzung einzufangen, ist gestiegen.

Gestrichen wurden im selben Zuge einige Übergangsregelungen, die sich nur auf den ersten Nachweiszeitraum von Juli 2004 bis Juni 2009 bezogen haben. Damit gibt es praktisch keine Ausnahmeregelungen mehr. Sogar die bisherige Möglichkeit, sich bereits absolvierte Fortbildungen vor Ablauf des fünfjährigen Nachweiszeitraums von der KV bestätigen zu lassen, gibt es nicht mehr. Derartige Meldungen sind nur noch an die Landesärztekammern möglich, sofern diese das anbieten.

\title{
Palliativmedizin: Im EBM drohen Einschränkungen
}

_ Im hausärztlichen Teil des EBM wurde zum 1. Oktober 2013 der Abschnitt 3.2.5 zur Förderung der palliativmedizinischen Versorgung neu aufgenommen. Er sieht sowohl abrechnungsfähige Leistungen zur Eingangsdiagnostik und palliativmedizinischen Ersterhebung als auch zur fortlaufenden palliativen Betreuung von Patienten vor. Die Zielgruppe der für die palliativmedizinische Versorgung vorgesehenen Patienten wird in der Einleitung des Abschnitts genau definiert.

Zusätzliche Qualifikationen müssen Hausärzte für die Abrechnung bisher nicht nachweisen. Die so geschaffene Abrechnungsmöglichkeit sollte das Augenmerk darauf richten, Palliativpatienten sowohl in der Praxis als auch in der eigenen Häuslichkeit adäquat zu versorgen.

\section{MMW-KOMMENTAR}

Seit Einführung der neuen palliativmedizinischen Abrechnungspositionen wird der strukturellen Trennung der Palliativmedizin in eine allgemeine ambulante Palliativversorgung (AAPV) und eine spezielle ambulante Palliativversorgung (SAPV) Rechnung getragen. Der Hausarzt kann sich jetzt entscheiden, ob er einen Palliativpatienten selbst, nur teilweise oder überhaupt nicht mehr behandelt und an ein Palliativteam überweist. Für letzteren Fall stehen die Nrn. 01425 und 01426 EBM zur Verfügung, mit der die Erstverordnung bzw. Folgeverordnung der spezialisierten ambulanten Palliativversorgung honoriert wird.

Leider werden die hausärztlichen Palliativleistungen viel zu selten abgerechnet, sodass die Einführung einer Zusatzweiterbildung als Abrechnungsvoraussetzung droht. Wer allerdings jetzt schon Palliativleistungen abrech- net, kann davon ausgehen, dass er im Falle einer künftigen Abrechnungseinschränkung von einer Übergangslösung profitiert. Oft übersehen wird bei der Versorgung von Palliativpatienten durch den Hausarzt nämlich, dass die dort in der Regel durchgeführte Ersterhebung im Krankheitsfall grundsätzlich nach Nr. 03370 abgerechnet werden kann - auch wenn der Patient an ein Palliativteam abgegeben wird.

Da aber mit einer solchen Weitergabe der Kontakt des Patienten und seiner Angehörigen zum Hausarzt oft nicht abbricht, sollte man überlegen, ob man auf der Verordnung nicht das Feld ", additiv unterstützende Teilversorgung" ankreuzt. In diesem Fall eröffnet sich bei Bedarf nämlich die Möglichkeit, Kontakte mit dem Patienten nach den Nrn. 03372 bzw. 03373 EBM zu berechnen. Das gilt insbesondere für Leistungen im Rahmen der häuslichen Versorgung 